\title{
Alcohol induces cell proliferation via hypermethylation of ADHFE1 in colorectal cancer cells
}

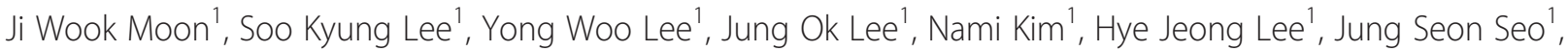 \\ Jin Kim², Hyeon Soo Kim ${ }^{1}$ and Sun-Hwa Park ${ }^{1 *}$
}

\begin{abstract}
Background: The hypermethylation of Alcohol dehydrogenase iron containing 1 (ADHFE1) was recently reported to be associated with colorectal cancer (CRC) differentiation. However, the effect of alcohol on ADHFE1

hypermethylation in CRC is still unclear.

Methods: The methylation status and expression levels of ADHFE1 were investigated in primary tumor tissues and adjacent normal tissues of 73 patients with CRC, one normal colon cell line, and 4 CRC cell lines (HT-29, SW480, DLD-1, and LoVo) by quantitative methylation-specific polymerase chain reaction (QMSP) and real-time reverse transcription polymerase chain reaction (real time PCR), respectively. The effect of alcohol on the methylation status of ADHFE1 was analyzed in HT-29, SW480, DLD-1, and CCD18Co cells using QMSP, real-time PCR, immunoblot, and cell proliferation assay.
\end{abstract}

Results: ADHFE1 was hypermethylated in 69 of 73 CRC tissues (95\%) compared to adjacent normal tissues $(p<0.05)$. The mRNA expression of ADHFE1 was significantly reduced in CRC compared to adjacent normal tissues $(p<0.05)$ and its expression was decreased in the alcohol consumption group $(p<0.05)$. ADHFE1 was hypermethylated and its expression was decreased in 4 CRC cell lines compared with normal colon cell line. Alcohol induced hypermethylation of ADHFE1, decreased its expression, and stimulated cell proliferation of HT-29, SW480, and DLD-1cells.

Conclusion: These results demonstrate that the promoter hypermethylation of ADHFE1 is frequently present in CRC and alcohol induces methylation-mediated down expression of ADHFE1 and proliferation of CRC cells.

Keywords: ADHFE1, Colorectal cancer, Hypermethylation, Alcohol, Quantitative methylation-specific polymerase chain reaction

\section{Background}

Aberrant DNA methylation leads to the suppression of tumor suppressor gene expression as an epigenetic events [1]. In mammals, DNA methylation occurs only at CpG dinucleotide pairs, in which a $5^{\prime}$ cytosine residue is situated adjacent to a guanine residue. Hypermethylation of CpG-rich or intermediate promoters has been shown to inactivate downstream gene expression, and promoter hypermethylation of tumor suppressor genes is frequently

\footnotetext{
* Correspondence: parksh@korea.ac.kr

'Institute of Human Genetics, Department of Anatomy, Korea University College of Medicine, 126-1, Anam-dong 5-ga, Seongbuk-gu, Seoul 136-705, Republic of Korea

Full list of author information is available at the end of the article
}

observed in many human malignancies, and may contribute to disease pathogenesis [2-5]. Colorectal cancer (CRC) is one of the most common cancers in Korea, and its incidence has been steadily increasing. Epigenetic alterations that are commonly found in CRCs include DNA methylation of tumor suppressor genes [6,7] and histone deacetylation [8].

Alcohol overconsumption is a well-known risk factor for the development as well as progression of various types of cancers, including CRC [9]. When alcohol is consumed, it is metabolized by alcohol dehydrogenases (ADH) and cytochrome P450 subenzyme 2E1 (CYP2E1), which catalyze the oxidation of alcohol to acetaldehyde [10]. Alcohol dehydrogenases $(A D H)$ are a well-defined 
group of enzymes involved in general detoxification of alcohols and are associated with several human cancers, including CRC [11]. Alcohol metabolism increases reactive oxygen species (ROS) generation, resulting in oxidative stress [12], and accumulation of ROS enhances arsenicinduced tumor angiogenesis in CRC cells via the HIF- $1 \alpha$ pathway [13]. Alcohol promotes cancer progression by inducing gene expression of epithelial-mesenchymal transition (EMT) genes, such as the transcription factor Snail, by increasing epidermal growth factor receptor (EGFR) transactivation and activating matrix metalloproteinases (MMPs) [14].

Alcohol dehydrogenase, iron containing, 1 (ADHFE1) located on chromosome 8q13.1 was cloned by Deng and colleagues from a human fetal brain cDNA library [15]. ADHFE1 is related to members of the group III metaldependent alcohol dehydrogenase family [16], and encodes hydroxyacid-oxoacid transhydrogenase, which is responsible for the oxidation of 4-hydroxybutyrate to succinate semialdehyde [17]. The hypermethylation of ADHFE1 was recently reported in CRC $[18,19]$ and is associated with differentiation [20]. However, the association between the hypermethylation of ADHFE1 and alcohol in CRC has not been reported yet.

In this study, the hypermethylation of ADHFE1 was identified in CRC using quantitative methylation-specific polymerase chain reaction (QMSP). The expression level of ADHFE1 in CRC tissues was compared to that in adjacent normal tissues using real-time reverse transcriptasepolymerase chain reaction (real-time PCR). We investigated the demethylating effects of ADHFE1 using 5-aza2 '-deoxycytidine. We analyzed the effect of alcohol on methylation and expression of ADHFE1 as well as cell proliferation in CRC cells.

\section{Methods}

\section{Tissues}

Fresh-frozen primary tumors $(n=73)$, paired tumors, and adjacent normal tissues $(n=73)$ from CRC patients were collected at the time of surgery at the Korea University Medical Center. The clinicopathologic features of CRC patients are summarized in Table 1. The "drinking group" comprised individuals consuming more than $300 \mathrm{~mL}$ of alcohol three or more times per a week and the "non-drinking" group contained individuals who did not consume alcohol. The tissues were collected after obtaining informed consent from the patients and the study was approved by the Institutional Review Board of Korea University (IRB No: KU-IRB-10-08-A-1). The diagnosis of CRC tissues was acquired from pathology reports histological evaluations.

\section{Cell lines}

One normal colon cell line (CCD18Co) and 4 CRC cell lines (HT-29; SW480, Dukes' type B; DLD-1, Dukes'
Table 1 Clinicopathologic characteristics of colorectal cancer patients and methylation status of ADHFE1

\begin{tabular}{|c|c|c|c|}
\hline \multirow[t]{2}{*}{ Characteristics } & \multirow[t]{2}{*}{ No. of cases } & \multicolumn{2}{|c|}{$\begin{array}{l}\text { Methylation status of } A D H F E 1 \\
\text { (PMR, \%) }\end{array}$} \\
\hline & & Median (range) & $p$-Value \\
\hline Normal & 73 & $3.25( \pm 0.50)$ & $<0.001^{\dagger}$ \\
\hline Colorectal cancer & 73 & $74.89( \pm 8.06)$ & \\
\hline Age (years) & & & 0.957 \\
\hline$\leq 65$ & 35 & $75.35( \pm 13.73)$ & \\
\hline$>65$ & 38 & $74.46( \pm 9.13)$ & \\
\hline Gender & & & 0.114 \\
\hline Female & 27 & $91.55( \pm 16.46)$ & \\
\hline Male & 46 & $65.10( \pm 8.21)$ & \\
\hline Location & & & 0.627 \\
\hline Colon & 46 & $77.91( \pm 10.45)$ & \\
\hline Rectum & 27 & $69.73( \pm 12.77)$ & \\
\hline TNM Stage & & & 0.252 \\
\hline$|| \mid$, & 32 & $85.40( \pm 14.10)$ & \\
\hline$\| I I, I V$ & 41 & $66.68( \pm 9.18)$ & \\
\hline Size (mm) & & & 0.276 \\
\hline$\leq 25$ & 44 & $67.71( \pm 7.81)$ & \\
\hline$>25$ & 29 & $85.78( \pm 16.48)$ & \\
\hline Alcohol consumption & & & $0.012^{\dagger}$ \\
\hline Non-drinking & 36 & $95.27( \pm 13.15)$ & \\
\hline Drinking & 37 & $55.06( \pm 8.41)$ & \\
\hline
\end{tabular}

Statistical significance is evaluated by analysis of variance (ANOVA). ${ }^{\dagger} p$-Values of $<0.05$ are considered statistically significant.

PMR: Percentage of methylated reference; TNM: Tumor, lymph nodes and metastasis.

type C; LoVo, Dukes' type C and stage IV) were obtained from the American Type Culture Collection (Manassas, VA, USA). CCD18Co cells were cultured in Eagle's minimum essential medium and the 4 CRC cells were cultured in RPMI 1640 medium, all supplemented with $10 \%$ fetal bovine serum (Hyclone, Logan, UT, USA) and $1 \%$ penicillin/streptomycin (P/S; Life Technologies). The cells were maintained at $37^{\circ} \mathrm{C}$ and $5 \%$ $\mathrm{CO}_{2}$ atmosphere.

\section{Genomic DNA extraction}

Genomic DNA was extracted using the QIAamp DNA Mini Kit (Qiagen, Valencia, CA, USA) according to the manufacturer's recommendations. Tissue samples were ground up by 3-mm diameter punches and then mixed with $700 \mu \mathrm{L}$ lysis buffer containing $20 \mu \mathrm{g} / \mathrm{mL}$ Labo Pass protease K (Cosmo Gene Tech., Seoul, Korea), $20 \mathrm{mM}$ Tris. $\mathrm{HCl}$ (pH 8.0), 5 mM EDTA (pH 8.0), $400 \mathrm{mM} \mathrm{NaCl}$, and $1 \%$ SDS solution (Sigma-Aldrich, St. Louis, MO, USA). The mixed samples were incubated at $42^{\circ} \mathrm{C}$ overnight. After incubation, genomic DNA was purified by phenol/chloroform extraction, eluted in $100 \mu \mathrm{L}$ of water, 
and quantified with a NanoDrop ND-100 device (Thermo Fisher Scientific, Hudson, NH, USA).

\section{Sodium bisulfite DNA modification}

Two micrograms of genomic DNA in $20 \mu \mathrm{L}$ of RNase-free water was bisulfite converted using the EpiTect fast DNA bisulfite kit (Qiagen) according to the manufacturer's recommendations. The reaction was performed by mixing $85 \mu \mathrm{L}$ of bisulfite mix solution and $35 \mu \mathrm{L}$ of DNA protect buffer in $200 \mu \mathrm{L}$ PCR tubes at room temperature. The bisulfite-converted genomic DNA was eluted from the column with $100 \mu \mathrm{L}$ of $\mathrm{dH}_{2} \mathrm{O}$ and stored at $-80^{\circ} \mathrm{C}$ until use.

\section{Quantitative methylation specific PCR (QMSP)}

Quantitative methylation status of the bisulfite-converted genomic DNA was confirmed by quantitative real-time PCR using the 7500 Real-Time PCR System (Applied Biosystems, San Francisco, CA, USA) according to the manufacturer's recommendations. Methylation primers were designed using the MethPrimer software (http:// www.urogene.org/methprimer/). MSP primer sequences for the methylated sequence of ADHFE1 ( -100 to +202 , position from translational start site +1$)$ : $5^{\prime}$ - AGG GCG GTA TTT AAA TTT TTC GAA TT -3' (sense), 5' - CGC GAA ACG AAT AAA CAA ACG CGA CCG A -3' (antisense) ); reference sequence of beta-actin (ACTB) (-1645 to -1513): 5' - TGG TGA TGG AGG AGG TTT AGT AAG T -3' (sense), 5' - AAC CAA TAA AAC CTA CTC CTC CCT TAA -3' (antisense). The product sizes were 303 bp and 132 bp respectively. PCR reactions were performed using an optical 96-well tray in a final volume of $20 \mu \mathrm{L}$. The reaction mixture consisted of $5 \mu \mathrm{L}$ of $2 \mathrm{X}$ Maxima SYBR Green/ROX qPCR master mix (Thermo Fisher Scientific), $250 \mathrm{nM}$ of each primer, and $100 \mathrm{ng}$ of bisulfiteconverted DNA template. The QMSP program was as the following: $95^{\circ} \mathrm{C}$ for $10 \mathrm{~min}$, followed by 45 cycles at $95^{\circ} \mathrm{C}$ for $15 \mathrm{~s}$, and then $60^{\circ} \mathrm{C}$ for $1 \mathrm{~min}$. After PCR, a thermal melt profile was performed to examine the homogeneity of the PCR application. Each DNA sample was analyzed in triplicate, and the mean quantity was used for further analysis. Relative quantification of the amplified gene levels in the bisulfite-converted genomic DNA sample was performed by measuring the threshold cycle $\left(\mathrm{C}_{\mathrm{T}}\right)$ values of $A D H F E 1$ and $\beta$-actin $(A C T B)$. The mean quantity of genes was divided by the mean quantity of $A C T B$ and was used for the normalization of input DNA. The negative values for $A C T B$ were excluded from the methylation analysis. The bisulfite-converted genomic DNA of a known concentration was prepared at $1,1 / 4$, $1 / 16$, and $1 / 64$ by serial dilutions, and used in a standard curve for quantification. The modified genomic DNA by CpG methyltransferase M.SssI (NEB, Ipswich, MA, USA) was used as a positive control according to the manufacturer's recommendations. DNA methylation according to M.SssI was verified using the restriction enzyme BstUI (NEB).

\section{mRNA extraction and cDNA synthesis}

mRNA was extracted using an RNeasy Mini kit (Qiagen) according to the manufacturer's recommendations. mRNA was eluted in $20 \mu \mathrm{L}$ of diethyl pyrocarbonate (DEPC) water (Qiagen) and quantified with a NanoDrop ND-100 device (Thermo Fisher Scientific). cDNA was synthesized from $1 \mu \mathrm{g}$ of mRNA from each sample using Moloney murine leukemia virus reverse transcriptase (M-MLV RT) and random hexamers (Promega, Madison, WI, USA). The cDNA synthesis reaction was prepared according to the manufacturer's recommendations by mixing $1 \mu \mathrm{g}$ mRNA, $4 \mu \mathrm{L}$ of $5 \times$ RT buffer, $1 \mu \mathrm{L}$ of $500 \mathrm{nM}$ oligo dT, $1 \mu \mathrm{L}$ of a $10 \mathrm{mM}$ dNTP solution, $0.5 \mu \mathrm{L}$ of RNasin, $1 \mu \mathrm{L}$ of M-MLV RT, and $12.5 \mu \mathrm{L}$ of distilled water in PCR tubes. The mixture was incubated in $37^{\circ} \mathrm{C}$ for $1 \mathrm{~h}$. cDNA was diluted with $20 \mu \mathrm{L}$ of distilled water and stored at $-80^{\circ} \mathrm{C}$ until use.

\section{Real-time PCR}

mRNA expression was confirmed by quantitative realtime PCR using a 7500 Real-Time PCR System (Applied Biosystems) according to the manufacturer's recommendations. The primers were designed using Primer3 version 0.4 .0 (http://primer3.ut.ee/). The specific primers were: ADHFE1: 5' - TGC CAT TTT TGA CTA TGA ACA CTT -3' (sense), 5' - GAC AGC CCT CTT CAG ATA CTT AGC -3'(antisense); ACTB, 5' - AGA GCT ACG AGC TGC CTG AC -3' (sense) and 5'- AGC ACT GTG TTG GCG TAC AG -3' (antisense. The product sizes of $A D H F E 1$ and $A C T B$ were 304 bp and 184 bp, respectively. The PCR reaction was performed in a final volume of $20 \mu \mathrm{L}$ using an optical 96-well tray. The reaction mixture consisted of $5 \mu \mathrm{L}$ of $2 \times$ Maxima $^{\oplus}$ SYBR Green/ROX qPCR Master Mix (Thermo Fisher Scientific), $250 \mathrm{nM}$ of each primer, and $100 \mathrm{ng}$ of cDNA template. The real-time PCR program was initiated at $95^{\circ} \mathrm{C}$ for $10 \mathrm{~min}$, followed by 35 cycles of $95^{\circ} \mathrm{C}$ for $15 \mathrm{~s}$ and $60^{\circ} \mathrm{C}$ for $1 \mathrm{~min}$. The thermal melt profile was examined to assess the homogeneity of the PCR application. Each DNA sample was analyzed in triplicate, and the mean quantity was used for further analysis. The relative levels of amplified mRNA in each sample were quantified by measuring the threshold cycle $\left(\mathrm{C}_{\mathrm{T}}\right)$ values of target genes. The mean quantity of each gene was divided by the mean quantity of $A C T B$ and was used for the normalization of input DNA. cDNA of a known concentration was prepared at 1, 1/10, $1 / 100$, and $1 / 1000$ by serial dilutions and used as the standard curve for quantification. 


\section{Chemical treatment}

To determine the optimal concentration of ethanol (Sigma-Aldrich) in a normal colon cell line and 4 CRC cell lines, we measured cell viability with the 3-(4,5dimethylthiazol-2-yl)-2,5-diphenyltetrazolium (MTT) assay (data not shown) according to the manufacturer's recommendations using MTT reagents $(10 \mu \mathrm{L} /$ well, $7.5 \mathrm{mg} / \mathrm{mL}$ in phosphate-buffered saline, PBS) and dimethyl sulfoxide (50 $\mu \mathrm{L} /$ well, Sigma-Aldrich). To identify the alteration of methylation status by treatment with ethanol, cells were seeded in 6-well culture plates (SPL LifeScience, Pocheon, Korea) at a density of $0.5 \times 10^{5}$ cells per well. After $24 \mathrm{~h}$, the cells were treated with $100 \mathrm{mM}$ ethanol for $72 \mathrm{~h}$ at $37^{\circ} \mathrm{C}$ in a $5 \% \mathrm{CO}_{2}$ atmosphere. The cells were washed in PBS three times and then harvested. The cells were seeded at a density of $0.5 \times 10^{5}$ cells/well in a 6 -well plate, and after $24 \mathrm{~h}$, the cells were treated with demethylation agent $30 \mu \mathrm{M} 5$-aza-2' -deoxycytidine (5-aza-dC) for $72 \mathrm{~h}$. The results were drawn from experiments repeated at least three times.

\section{ADHFE1 siRNA treatment}

CCD18Co and DLD-1 cells were seeded at a density of $0.5 \times 10^{5}$ cells/well in a 6-well plate and allowed to grow to $70 \%$ confluence for $24 \mathrm{~h}$. Cells were serum-starved for 30 min before siRNA transfection. Transient transfections were performed using a transfection reagent (Lipofectamine 2000; Life Technologies, Carlsbad, CA, USA) according to the manufacturer's protocol. A commercial ADHFE1 siRNA was purchased from Qiagen. The specific primers were: ADHFE1 siRNA: 5' - GGA UGU UGA UGA UGG CCU ATT -3' (sense), 5' - UAG GCC AUC AUC AAC AUC CAG -3' (anti-sense); non-target siRNA: 5' UUC UCC GAA CGU GUC ACG UTT -3' (sense), 5' ACG UGA CAC GUU CGG AGA ATT -3' (anti-sense). Fifty $\mathrm{nM}$ of siRNA and $6 \mu \mathrm{L}$ of transfection reagent were each diluted first with $100 \mu \mathrm{L}$ of reduced serum media, and then mixed. The mixtures were allowed to incubate for 10 minutes at room temperature and then added dropwise to each culture well containing $1 \mathrm{~mL}$ of reduced serum media. After $4 \mathrm{~h}$, the medium was changed using fresh complete medium. Cells were cultivated for $24 \mathrm{~h}$, $48 \mathrm{~h}$, or $72 \mathrm{~h}$. The cells were washed in PBS (SigmaAldrich) three times and then harvested.

\section{Cell counting assay and cell image capture}

After treatment with indicated agents, CCD18Co and DLD-1 cells in 6-well plates were washed with PBS (Sigma-Aldrich). The cells were detached with $0.05 \%$ trypsin (Wellgene, Deagon, Korea) and $0.53 \mathrm{mM}$ EDTA (Wellgene, Deagon, Korea) for $2 \mathrm{~min}$ at $37^{\circ} \mathrm{C}$. Eight hundred microliters of media supplemented with $10 \%$ FBS (Hyclone) was then added, and $20 \mu \mathrm{L}$ of cells were combined with $20 \mu \mathrm{L}$ of $0.4 \%$ trypan blue solution
(Sigma-Aldrich). The retained cells were transferred to a counting chamber (Paul Marienfeld GmbH \& Co. KG, Lauda-Königshofen, Germany) and cells were counted using Nikon TMS inverted stage microscope (Nikon Instrument Inc., Melville, NY, USA). For counter staining, cells were fixed with $4 \%$ paraformaldehyde (SigmaAldrich) for $15 \mathrm{~min}$ at room temperature, and the fixed cells were washed three times in PBS (Sigma-Aldrich). Cells were stained with Hoechst 33342 (Sigma-Aldrich) for $10 \mathrm{~min}$ at room temperature without light and washed with PBS (Sigma-Aldrich). Images of cells were obtained using a LSM 700 Confocal Laser Scanning Microscope (Carl Zeiss Co., Ltd., Jena, Germany). The results were drawn from experiments repeated at least three times.

\section{Immunoblot analysis}

Cells were grown in 6-well plates and treated with the indicated agents. Following treatment, the media was aspirated and the cells were washed three times in ice-cold PBS (Sigma-Aldrich) and lysed in $100 \mu \mathrm{L}$ of lysis buffer. The samples were then briefly sonicated, centrifuged for $5 \mathrm{~min}$, and supernatants were boiled for $5 \mathrm{~min}$ at $95^{\circ} \mathrm{C}$. The supernatants were subjected to electrophoresis on sodium dodecyl sulfate polyacrylamide gel electrophoresis (10\%) gels, and transferred to polyvinylidene difluoride membranes. The blots were incubated overnight at room temperature with primary ADHFE1 antibody (Sigma-Aldrich), and then washed six times in Trisbuffered saline/0.1\% Tween 20 prior to incubation with horseradish peroxidase-conjugated secondary antibody (anti-rabbit, Sigma-Aldrich) for $1 \mathrm{~h}$ at room temperature. The blots were visualized using ECL (Amersham Biosciences, Buckinghamshire, UK). Beta-actin $(A C T B)$ and glyceraldehyde-3-phosphate dehydrogenase (GAPDH) were used as loading controls.

\section{Fluorescence-activated cell sorting (FACS) analysis}

Cells treated with propidium iodide (PI) can be utilized not only for assessment of the stages of the cell cycle (G0/G1, S, G2/M), but also for identification of apoptotic cells (hypodiploid, sub G0 peak). Half a million cells were pelleted at $1000 \times \mathrm{g}$ for $5 \mathrm{~min}$ and were mixed with $10 \mu \mathrm{l}$ of Vindelov's PI (Sigma-Aldrich) stain solution (1.21 g TRIS base, $584 \mathrm{mg} \mathrm{NaCl}, 10 \mathrm{mg}$ RNAse, $50.1 \mathrm{mg}$ PI, $1 \mathrm{ml}$ Nonidet P40, $\mathrm{pH} \mathrm{8.0)}$ and $490 \mu \mathrm{l}$ of standard azide buffer(PBS buffer, 0.5\% EDTA pH 8.0). Cells were incubated at $4{ }^{\circ} \mathrm{C}$ overnight and analyzed using an FACS caliber flow cytometer (Becton-Dickinson, NJ, USA) at an excitation wavelength of $488 \mathrm{~nm}$ and an emission wavelength of $525 \mathrm{~nm}$. The 10,000 cells were measured by gating the polymorphonuclear leukocytes (PMN) population and analyzed using the BD FACSDiva Software version 6. 1. 3 (Becton-Dickinson). 


\section{Statistical analysis}

MTT assay results were quantified using SoftMax ${ }^{\bullet}$ Pro software (Molecular Devices, LLC). The methylated intensity ratio of QMSP was determined as the percentage of methylated reference (PMR), and the PMR value was defined as: $\left[(A D H F E 1)_{\text {sample }} /(A C T B)_{\text {sample }}\right] /$ $\left[(A D H F E 1)_{\mathrm{M} . S s s} /(A C T B)_{\mathrm{M} . S s s}\right] \times 100$. A PMR value of ten or more indicated hypermethylation. The significance of the differences in PMR values was defined by the chi-squared test, Fisher's exact test, and ANOVA using Sigma Stat (SPSS Inc., Chicago, IL, USA). In all statistical tests, $p$-Values of $<0.05$ were considered statistically significant. Real-time PCR and immunoblot analysis data were compared and qualitative differences between samples were analyzed using Sigma Stat (SPSS Inc.). $p$-Values of $<0.05$ were considered statistically significant.

\section{Results}

ADHFE1 is hypermethylated and down regulated in CRC To determine the methylation status of ADHFE1, we analyzed the methylation status of $73 \mathrm{CRC}$ tissues and adjacent normal tissues using QMSP. ADHFE1 was hypermethylated in 69 out of 73 CRC tissues (95\%) and only 2 out of 73 adjacent normal tissues (3\%). Comparative analysis using percentage of methylated reference (PMR) value also indicated that the methylation status of ADHFE1 was much higher in CRC tissues compared to adjacent normal tissues (Figure $1 \mathrm{~A}$ ). To verify whether the mRNA expression of ADHFE1 is caused by aberrant DNA methylation, we examined the expression level of ADHFE1 in 73 CRC tissues and adjacent normal tissues by real-time PCR. The mRNA expression of ADHFE1 was significantly reduced in CRC tissues compared to adjacent normal tissues (Figure 1B). We performed statistical analysis between the methylation of ADHFE1 and clinicopathologic features of CRC. The PMR value of ADHFE1 was not significantly different in most clinicopathologic features except the alcohol consumption in CRC tissues (Table 1). The methylation status of ADHFE1 in CRC tissues was much higher in the nondrinking group than the drinking group $(p<0.05$, Figure $1 C$ ). However, mRNA expression of ADHFE1 in CRC tissues, compared to adjacent normal tissues, was more reduced in groups of drinking and old age (Figure 1D, Table 2).
A

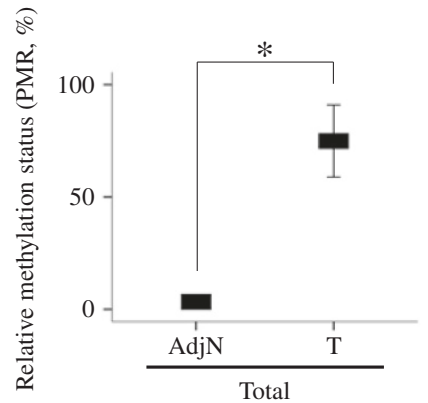

$\mathrm{C}$

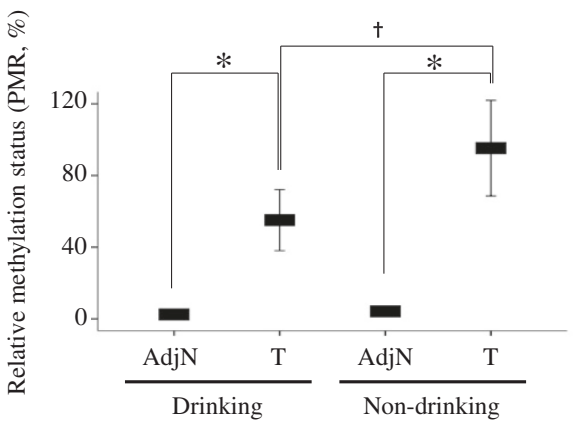

B

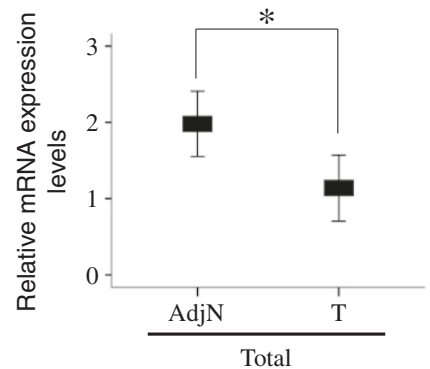

$\mathrm{D}$

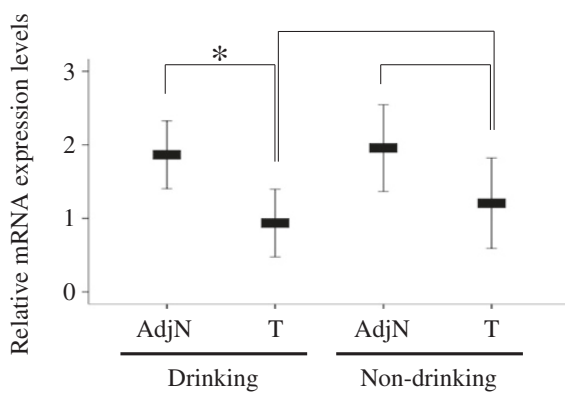

Figure 1 The methylation status and mRNA expression levels of ADHFE1 in CRC tissues and adjacent normal tissues. The methylation status of ADHFE1 in 73 CRC tissues and adjacent normal tissues is assessed using QMSP. A, C. ADHFE1 is hypermethylated in CRC (A) and also significantly hypermethylated in drinking groups and non-drinking groups (C). B. The expression levels of ADHFE1 in 73 CRC tissues compared with adjacent normal tissues was determined in 73 CRC tissues by real-time PCR. The mRNA expression of ADHFE1 is significantly decreased in CRC compared to adjacent normal tissues. D. The down regulation of $A D H F E 1$ is presented in drinking groups and non-drinking groups. ${ }^{*}, t p$-Values of $<0.05$ were considered statistically significant. AdjN: Adjacent normal tissue; T: Colorectal cancer tissues; PMR;: Percentage of methylated reference. 
Table 2 mRNA expression of ADHFE1 associated with CRC risk factors

\begin{tabular}{|c|c|c|c|}
\hline \multirow[t]{3}{*}{ Characteristics } & \multicolumn{2}{|c|}{ mRNA expression of $A D H F E 1$} & \multirow[t]{3}{*}{$p$-value } \\
\hline & $\overline{\operatorname{AdjN}}$ & $\mathrm{T}$ & \\
\hline & $\overline{\text { Median (range) }}$ & $\overline{\text { Median (range) }}$ & \\
\hline Colorectal cancer & $1.98( \pm 0.21)$ & $1.14( \pm 0.22)$ & $0.007^{\dagger}$ \\
\hline \multicolumn{4}{|l|}{ Age (years) } \\
\hline$\leq 65$ & $1.97( \pm 0.52)$ & $1.54( \pm 0.41)$ & 0.525 \\
\hline$>65$ & $1.94( \pm 0.29)$ & $1.04( \pm 0.30)$ & $0.034^{\dagger}$ \\
\hline \multicolumn{4}{|c|}{ Alcohol consumption } \\
\hline Non-drinking & $1.96( \pm 0.29)$ & $1.21( \pm 0.30)$ & 0.078 \\
\hline Drinking & $1.87( \pm 0.23)$ & $0.94( \pm 0.23)$ & $0.005^{\dagger}$ \\
\hline
\end{tabular}

Statistical significance is evaluated by analysis of variance (ANOVA). ${ }^{+} p$-Values of $<0.05$ are considered statistically significant.

AdjN: Adjacent normal tissue; T: Colorectal cancer tissues.

Restoration of ADHFE1 expression by 5 -aza-dC in CRC cells The effect of methylation on mRNA expression was determined by MSP and real-time PCR analysis in 5-aza-dC treated HT-29, SW-480, DLD-1, LoVo, and CCD18Co cells. ADHFE1 was hypermethylated in 4 CRC cells and demethylated by treatment with 5-aza-dC (Figure 2A).
The mRNA expression of ADHFE1 was relatively reduced in 4 CRC cells compared with CCD18Co and was restored by treatment with 5 -aza-dC (Figure $2 \mathrm{~B}$ ). To confirm the protein expression of ADHFE1, we measured ADHFE1 protein levels and estimated the change in protein expression by 5 -aza-dC in all cell lines using western blot analysis. The basal protein level of ADHFE1 was lower in 4 CRC cells compare to that of CCD18Co cells, and protein expression of ADHFE1 in 4 CRC cells increased upon treatment with 5 -aza-dC (Figure $2 \mathrm{C}$ ). These results suggest that promoter methylation of $A D H F E 1$ regulates the expression of $A D H F E 1$.

\section{Alcohol down-regulates the expression of ADHFE1 by methylation in CRC cells}

To examine the correlation between ADHFE1 methylation and expression in the presence and absence of alcohol, the methylation status and mRNA levels of ADHFE1 were assessed by QMSP and real-time PCR analysis, respectively, in 3 CRC cells after ethanol treatment. The methylation status of ADHFE1 was significantly induced in SW480 and DLD-1 cell (Figure 3A), whereas its expression was decreased in HT-29, SW480, and DLD-1 cells by
A

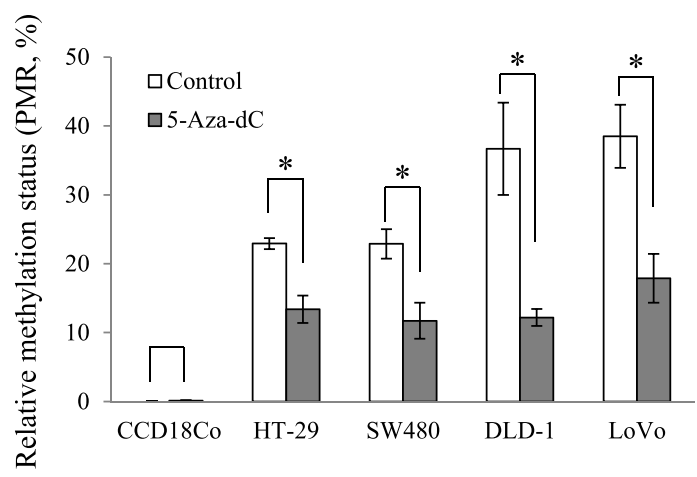

B

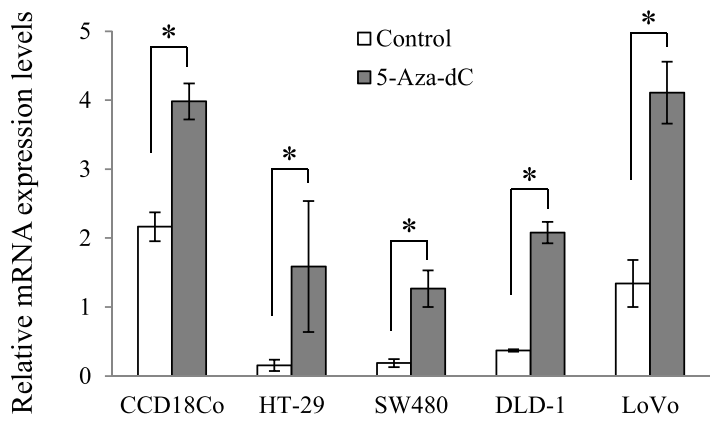

$\mathrm{C}$

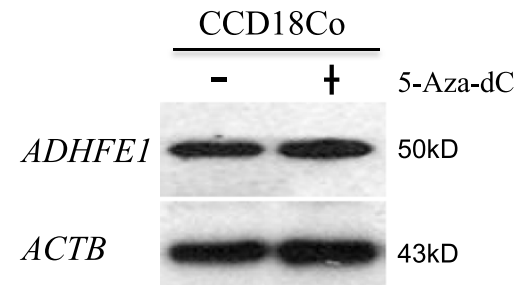

$\mathrm{D}$

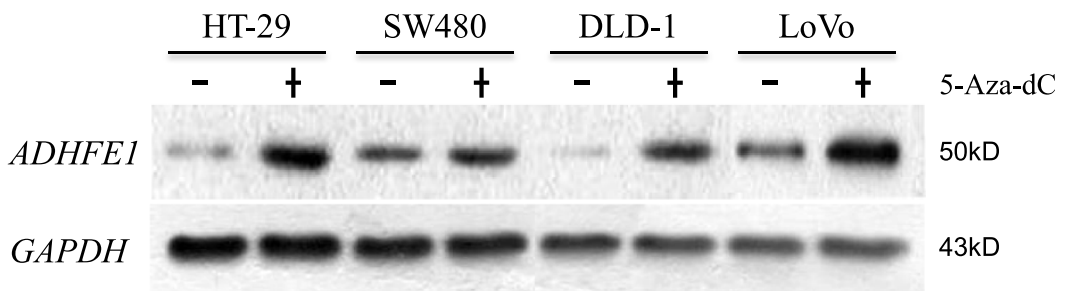

Figure 2 Changes of ADHFE1 methylation and expression by treatment with $\mathbf{5}$-aza-dC in CRC cells and normal colon cells. After treatment with 5-aza-dC in cells, the methylation status and expression levels of ADHFE1 are observed using QMSP, real-time PCR and immuno-blotting analysis. A. ADHFE1 is hypermethylated in 4 CRC cells compared to normal colon cells and significantly demethylated in 4 CRC cells by 5 -aza-dC. B. The mRNA expression of ADHFE1 is reduced in 4 CRC cells but increased in all cells treated with 5 -aza-dC. $\mathbf{C}$. The protein expression of $A D H F E 1$ is not affected by 5-aza-dC in CCD18Co. D. Protein expression of ADHFE1 is restored by 5-aza-dC in 4 CRC cells. Expression of ACTB and GAPDH is used as a loading control. * $p$-Values of $<0.05$ were considered statistically significant. +: Treated with 5-aza-dC; -: Non-treated with 5-aza-dC; PMR: Percentage of methylated reference. 


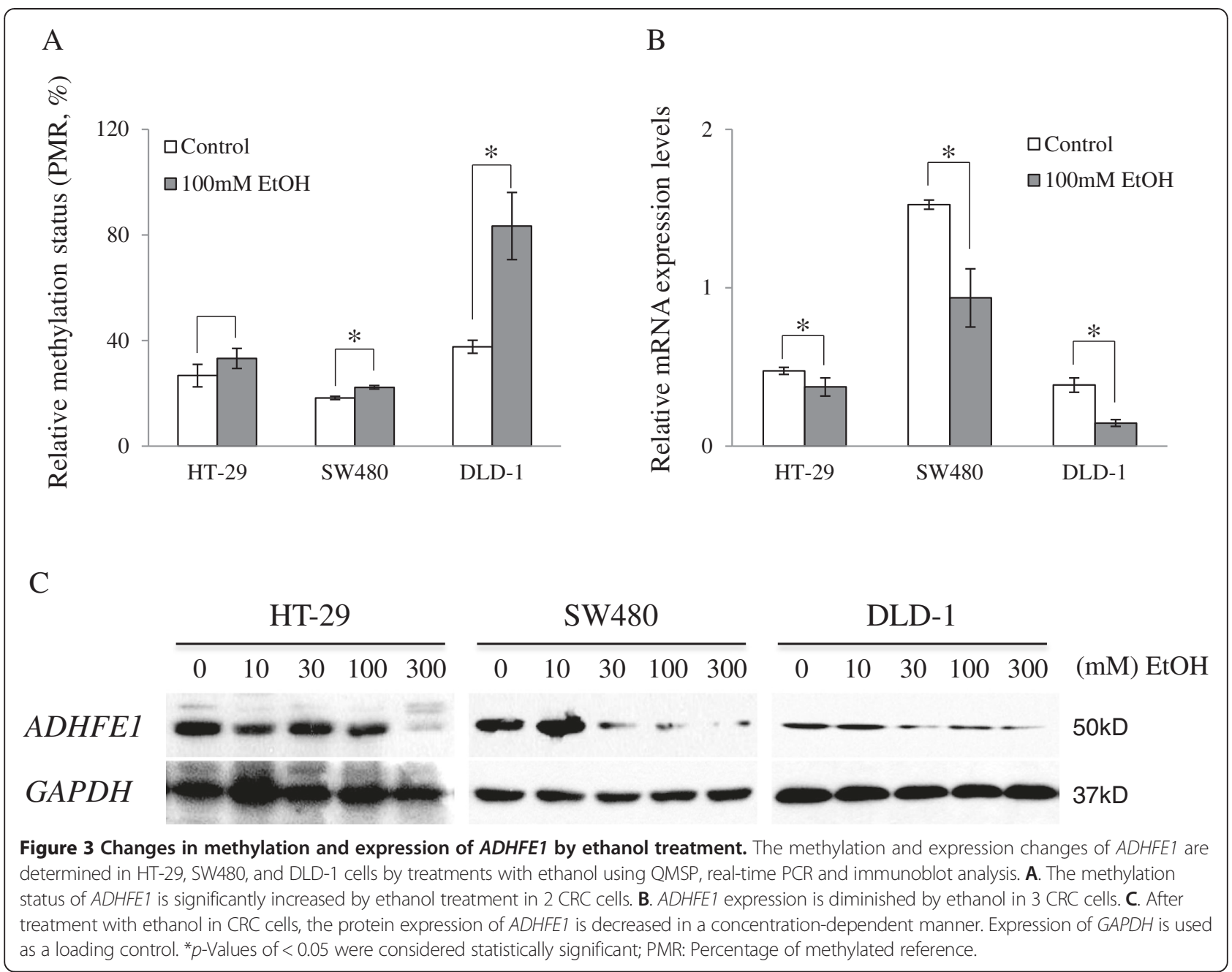

treatment with $100 \mathrm{mM}$ ethanol for 3 days (Figure 3B). In addition, we investigated the protein levels of ADHFE1 in 3 CRC cells after treatment with various concentrations of ethanol for 3 days. After treatment with ethanol, the protein expression of ADHFE1 was decreased in a concentration-dependent manner in both cells (Figure $3 \mathrm{C}$ ). These results suggest that alcohol promotes the hypermethylation of ADHFE1 and methylationmediated silencing of $A D H F E 1$.

\section{Alcohol induces the proliferation of CRC cells and down-expression of ADHFE1}

To investigate the function of $A D H F E 1$, its expression was inhibited by treatment with ethanol and transfection of ADHFE1 siRNA in HT-29, SW480, and DLD-1 cells. Cell viability and cell proliferation were then analyzed using MTT and a cell counting assay. The cell viability of HT-29, SW480, and DLD-1 cells was significantly increased by treatment with a combination of ethanol and ADHFE1 siRNA. HT-29 cells was increased the cell viability by ethanol as much as co-treatment (Figure 4A).
The cell counting assay showed that proliferation of 3 CRC cells was increased by ethanol and siRNA. Growth of DLD-1 cells by co-treatment was significantly higher than each treatment with alcohol and siRNA (Figure 4B). To confirm the effect of ADHFE1 down regulation on cell proliferation, cells were stained with Hoechst 33342 after ethanol treatment and siRNA transfection. The number of HT-29, SW480, and DLD-1 cells was increased by ethanol, siRNA, and co-treatment (Figure 4C). Furthermore, mRNA and protein expression of ADHFE1 were significantly decreased in $3 \mathrm{CRC}$ cells by treatment with alcohol, siRNA, and co-treatment (Figure 4D, 4E). These results suggest that methylation-mediated down regulation of ADHFE1 by alcohol may be associated with cell proliferation of CRC cells.

\section{Discussion}

Recently, aberrant methylation of ADHFE1 promoter was identified by a genome-wide methylation profiling screen using an array-based chip assay [18,19], and hypermethylation was found to be associated with CRC 


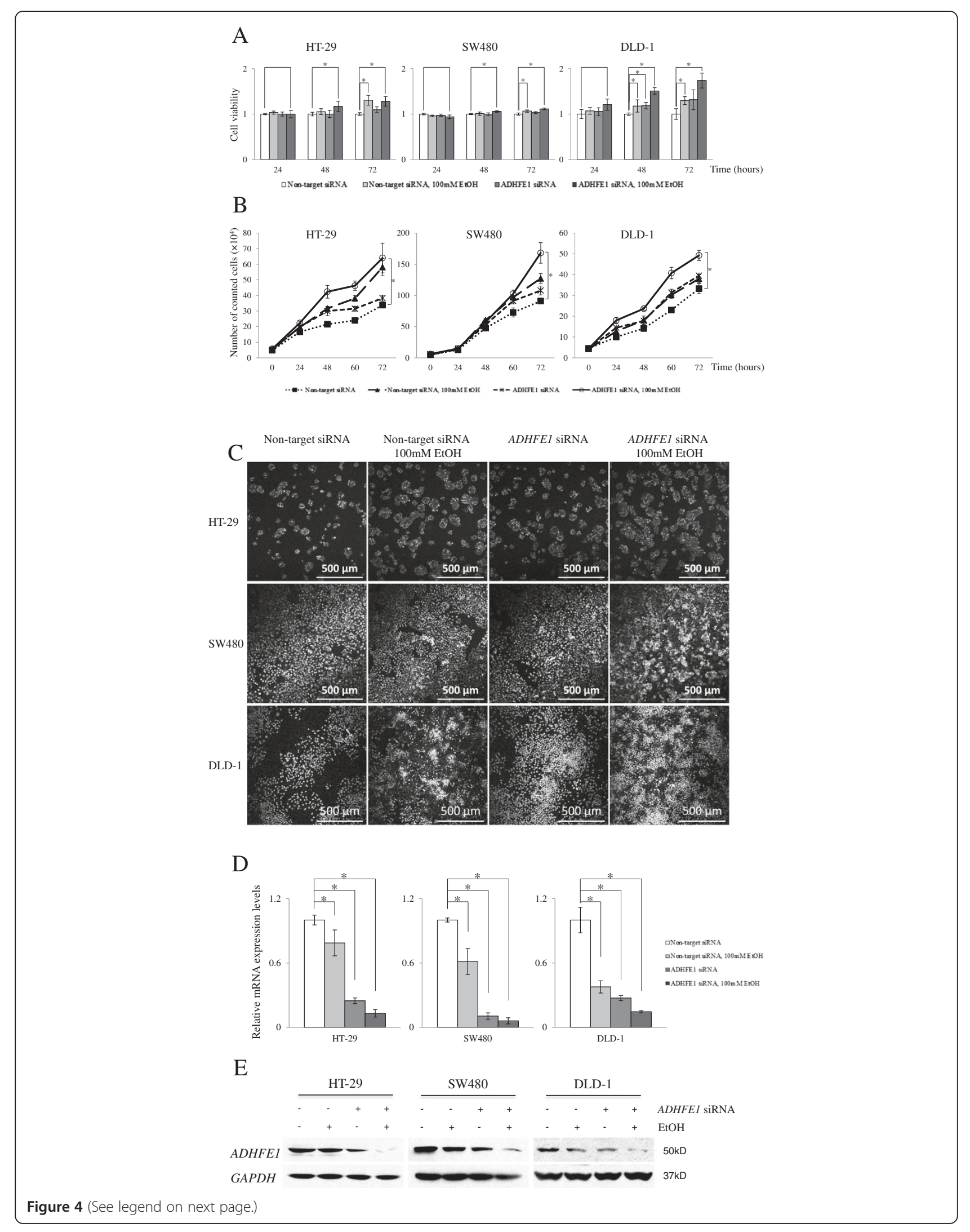


(See figure on previous page.)

Figure 4 The effect of ADHFE1 down regulation on cell viability and proliferation. The cell viability and proliferation of HT-29, SW480, and DLD-1 cells after ethanol treatment, transfection of ADHFE1 siRNA and combined treatment are determined by MTT, cell counting, and counter staining assay. A. Cell viability of 3 CRC cells is significantly increased by treatment with ethanol, siRNA, and combination of both. B. Cell proliferation of 3 CRC cells is significantly increased by ethanol, siRNA, and co-treatment. C. The captured images of cells using Hoechst 33342 show that the number of 3 CRC cells is increased by ethanol, siRNA, and co-treatment. $\mathbf{D}$. The mRNA expression of ADHFE1 is significantly decreased in 3 CRC cells by treatment with ethanol, siRNA, and combination of both. E. ADHFE1 protein expression is decreased in 3 CRC cells by treatment with ethanol, siRNA, and combination of both. GAPDH was used as a loading control. ${ }^{*} p$-Values of $<0.05$ were considered statistically significant. + : Treated with agent; -: Non-treated with agent.

differentiation [20]. In this study, we also identified the hypermethylation of ADHFE1 in CRC tissues compared to adjacent normal tissues using QMSP analysis. The PMR value of methylation status by QMSP was dichotomized at 4 PMR or 10 PMR for statistical purposes, as described previously [21,22]. In our experiments, 10 PMR or higher was considered as a cutoff to indicate a methylated state, whereas less than 10 PMR was considered as a cutoff to indicate an unmethylated state. Interestingly, the methylation status of ADHFE1 in CRC tissues was higher in the non-drinking group than in the drinking group, but the expression of ADHFE1 in CRC tissues compared to adjacent normal tissues was lower in the drinking group (Figure 1). The inverse correlation between ADHFE1 methylation and expression was highly significant in the drinking group. Based on this result, promoter methylation of $A D H F E 1$ may be the main factor affecting its expression in the drinking group.

The age-associated hypermethylation and methylationmediated silencing of several genes was reported in human prostate [23], colon tissues [24] and peripheral blood [25]. Based on the results presented here, we found no age-dependent ADHFE1 methylation but we identified that $A D H F E 1$ down regulation in CRC tissues compared to adjacent normal tissues was more significant in the old age group than the young age group. The inverse correlation between methylation and expression of ADHFE1 in 9 out of 12 CRC cell lines by 5 -aza-dC was reported by Tae C.H. et al. [20]. In this study, we obtained the same results in 4 CRC cell lines (Figure 2). This result suggests that the promoter methylation of $A D H F E 1$ may be the key regulator of $A D H F E 1$ expression in CRC cells.

Alcohol is a major risk factor well known for the progression of CRC $[10,26]$ and alters the methylation status of genes. Hypermethylation of alpha synuclein was significantly presented in peripheral blood of patients with chronic alcoholism [27]. Recently, array-based study has reported that various genes were hypermethylated in peripheral blood of patients with alcohol dependency compared to healthy controls [28]. We found that alcohol induced the hypermethylation of ADHFE1 and decreased its expression in CRC cells as well as normal colon cells. Down regulation of $A D H F E 1$ by siRNA affected cell growth in CRC cells. The survival rate and cell growth of SW480 and DLD-1 cells were significantly increased by treatment with a combination of ethanol and ADHFE1 siRNA than each individual treatment (Figure 4). The result demonstrates a synergetic effect between ethanol and ADHFE1 siRNA. The viability of HT-29 cells was affected more by the treatment with ethanol than down expression of $A D H F E$. In addition, we confirmed the viability of CRC cells after co-treatment with 5-aza-dC and ethanol. The viability of CRC cells was significantly decreased after treatment with 5-aza-dC. On the other hand, co-treated cells had smaller range of decline compared with 5-aza$\mathrm{dC}$ treated cells in HT-29 and SW480. The viability of DLD-1 cells were not affected by co-treatment (Additional file 1: Figure S1). In contrast, the down regulation of ADHFE1 by ethanol and siRNA had opposite effects on the viability and proliferation of CCD18Co, normal colon fibroblast cells (Additional file 2: Figure S2). CCD18Co was increased rate of apoptosis by treatment with ethanol, siRNA, and combination of both, but DLD-1 was not affected (Additional file 3: Figure S3). Previous studies have found that several genes, including interleukin 6 and tumor necrosis factor-alpha, have opposite functions in normal and tumor cells $[29,30]$. Taken together, our results provide evidence for different functions of ADHFE1 in normal colon cells and CRC cells.

\section{Conclusions}

We found that ADHFE1 was hypermethylated in CRC tissues compared to adjacent normal tissues and that the expression of ADHFE1 was significantly reduced in the alcohol drinking and old age group. In addition, alcohol induced the hypermethylation of ADHFE1 and decreased its expression in HT-29, SW480, and DLD-1 cells. The down regulation of $A D H F E 1$ by alcohol and ADHFE1 siRNA induced the growth of 3 CRC cells, and co-treatment with alcohol and siRNA greatly increased cell proliferation in SW480 and DLD-1 cells. These results suggest that hypermethylation of the ADHFE1 promoter by alcohol leads to a decrease in ADHFE1 expression and methylation-mediated silencing of $A D H F E 1$ may be induced the progression of CRC cells. 


\section{Additional files}

Additional file 1: Figure S1. The effect of ethanol, 5-aza-dC and co-treatment on the viability of CRC cells. The viability of HT-29, SW480, and DLD-1 cells after treatment ethanol, 5-aza-dC, and combination of both is determined by MTT assay. The viability of HT-29, SW480, and DLD-1 cells is reduced by treatment with 5-aza-dC. However, viability of HT-29 and SW480 cells treated with both 5-aza-dC and ethanol has smaller range of decline, compared to those treated with 5 -aza-dC. The viability of DLD-1 cells is not affected by co-treatment with 5 -aza-dC and ethanol. *Indicates the increase in cell viability by treatment with agent. HIndicates the decrease in cell viability by treatment with agent.

Additional file 2: Figure S2. The effect of ADHFE1 down regulation on cell viability and proliferation in normal colon cells. The cell viability and proliferation of CCD18Co after ethanol treatment, transfection of ADHFE1 siRNA, and combined treatment are determined by MTT, cell counting, and counter staining assay. A. The viability of CCD18Co cells is significantly decreased by ethanol, siRNA, and co-treatment. B. The proliferation of CCD18Co cells is significantly decreased by ethanol, siRNA, and cotreatment. C. The captured images of CCD18Co using Hoechst 33342 show that the number of CCD18Co cells is decreased by ethanol, siRNA, and co-treatment. D. ADHFE1 protein expression is decreased in CCD18Co cells treated with ethanol, siRNA, and combination of both. GAPDH was used as a loading control. * $p$-Values of $<0.05$ were considered as statistically significant. +: Treated with agent; -: Treated without agent.

Additional file 3: Figure S3. The effect of ADHFE1 down regulation on apoptosis in CCD18Co and DLD-1 cells. Apoptosis of CCD18Co and DLD-1 after ethanol treatment, transfection of ADHFE1 siRNA, and combined treatment is determined by FACS analysis. Apotosis of CCD18Co cells is induced by ethanol, ADHFE1 siRNA, and co-treatment, but that of DLD-1 cell are not affected.

\section{Abbreviations}

ACTB: Beta-actin; ADH: Alcohol dehydrogenase; ADHFE1: Alcohol dehydrogenase, iron containing, 1; CRC: Colorectal cancer; CYP2E1: Cytochrome P450 subenzyme 2E1; EGFR: Epidermal growth factor receptor; EMT: Epithelial-mesenchymal transition; MMPs: Matrix metalloproteinases; FACS: Fluorescence-activated cell sorting; GAPDH: glyceraldehyde-3-phosphate dehydrogenase; MTT: 3-(4,5dimethylthiazol-2-yl)-2,5-diphenyltetrazolium; QMSP: Quantitative methylation specific polymerase chain reaction; PBS: Phosphate-buffered saline; Real-time PCR: Real-time reverse transcription polymerase chain reaction; ROS: Reactive oxygen species; SAM: S-adenosylmethionine; TNM: Tumor, lymph nodes and metastasis; 5-aza-dC: 5-aza-2'-deoxycytidine.

\section{Competing interests}

The authors declare to have no competing interests.

\section{Authors' contributions}

JWM designed the study and drafted the manuscript. SKL and YWL prepared the clinical specimens and participated in the organization of clinical data. JOL, NK, and HJL participated in the analysis of in vitro analysis. JSS helped with the organization of the results. JK provided the clinical specimens and clinicopathologic information. HSK helped with the analysis of the results and revised the manuscript. SHP supervised the study and revised the manuscript. All authors have read and approved the final manuscript.

\section{Acknowledgements}

This study was supported by a Korea University Grant (K1132261) and the Basic Science Research Program through the National Research Foundation of Korea (NRF) funded by the Ministry of Education, Science and Technology (grant number: 2010-0024117).

\section{Author details}

1 Institute of Human Genetics, Department of Anatomy, Korea University College of Medicine, 126-1, Anam-dong 5-ga, Seongbuk-gu, Seoul 136-705, Republic of Korea. ${ }^{2}$ Department of General Surgery, Korea University Medical Center, 126-1, Anam-dong 5-ga, Seongbuk-gu, Seoul 136-705, Republic of Korea.
Received: 7 January 2014 Accepted: 16 May 2014

Published: 28 May 2014

\section{References}

1. Baylin SB, Herman JG: DNA hypermethylation in tumorigenesis: epigenetics joins genetics. Trends Genet 2000, 16:168-174.

2. Gao Y, Guan M, Su B, Liu W, Xu M, Lu Y: Hypermethylation of the RASSF1A gene in gliomas. Clin Chim Acta 2004, 349:173-179.

3. Goel A, Arnold CN, Tassone P, Chang DK, Niedzwiecki D, Dowell JM, Wasserman L, Compton C, Mayer RJ, Bertagnolli MM, Boland CR: Epigenetic inactivation of RUNX3 in microsatellite unstable sporadic colon cancers. Int J Cancer 2004, 112:754-759.

4. Lindsey JC, Lusher ME, Strathdee G, Brown R, Gilbertson RJ, Bailey S, Ellison DW, Clifford SC: Epigenetic inactivation of MCJ (DNAJD1) in malignant paediatric brain tumours. Int J Cancer 2006, 118:346-352.

5. Maruyama R, Sugio K, Yoshino I, Maehara Y, Gazdar AF: Hypermethylation of FHIT as a prognostic marker in nonsmall cell lung carcinoma. Cancer 2004, 100:1472-1477.

6. Fang JY, Chen YX, Lu J, Lu R, Yang L, Zhu HY, Gu WQ, Lu LG: Epigenetic modification regulates both expression of tumor-associated genes and cell cycle progressing in human colon cancer cell lines: Colo-320 and SW1116. Cell Res 2004, 14:217-226.

7. $\mathrm{Xu}$ L, Jain RK: Down-regulation of placenta growth factor by promoter hypermethylation in human lung and colon carcinoma. Mol Cancer Res 2007, 5:873-880

8. Chen YX, Fang JY, Lu J, Qiu DK: Regulation of histone acetylation on the expression of cell cycle-associated genes in human colon cancer cell lines. Zhonghua Yi Xue Za Zhi 2004, 84:312-317.

9. Boffetta P, Hashibe M: Alcohol and cancer. Lancet Oncol 2006, 7:149-156.

10. Haas SL, Ye W, Lohr JM: Alcohol consumption and digestive tract cancer. Curr Opin Clin Nutr Metab Care 2012, 15:457-467.

11. Salaspuro M: Acetaldehyde and gastric cancer. J Dig Dis 2011, 12:51-59.

12. Testino G: The burden of cancer attributable to alcohol consumption. Maedica 2011, 6:313-320.

13. Wang L, Son YO, Ding S, Wang X, Hitron JA, Budhraja A, Lee JC, Lin Q, Poyil $P$, Zhang $Z$, Luo J, Shi $X$ : Ethanol enhances tumor angiogenesis in vitro induced by low-dose arsenic in colon cancer cells through hypoxiainducible factor 1 alpha pathway. Toxicol Sci 2012, 130:269-280.

14. Forsyth CB, Tang Y, Shaikh M, Zhang L, Keshavarzian A: Alcohol stimulates activation of Snail, epidermal growth factor receptor signaling, and biomarkers of epithelial-mesenchymal transition in colon and breast cancer cells. Alcohol Clin Exp Res 2010, 34:19-31.

15. Deng Y, Wang Z, Gu S, Ji C, Ying K, Xie Y, Mao Y: Cloning and characterization of a novel human alcohol dehydrogenase gene (ADHFe1). DNA Seq 2002, 13:301-306.

16. Reid MF, Fewson CA: Molecular characterization of microbial alcohol dehydrogenases. Crit Rev Microbiol 1994, 20:13-56.

17. Kardon T, Noel G, Vertommen D, Schaftingen EV: Identification of the gene encoding hydroxyacid-oxoacid transhydrogenase, an enzyme that metabolizes 4-hydroxybutyrate. FEBS Lett 2006, 580:2347-2350.

18. Kim YH, Lee HC, Kim SY, Yeom Yl, Ryu KJ, Min BH, Kim DH, Son HJ, Rhee PL, Kim JJ, Rhee JC, Kim HC, Chun HK, Grady WM, Kim YS: Epigenomic analysis of aberrantly methylated genes in colorectal cancer identifies genes commonly affected by epigenetic alterations. Ann Surg Oncol 2011, $18: 2338-2347$

19. Oster B, Thorsen K, Lamy P, Wojdacz TK, Hansen LL, Birkenkamp-Demtroder K, Sorensen KD, Laurberg S, Orntoft TF, Andersen CL: Identification and validation of highly frequent $\mathrm{CpG}$ island hypermethylation in colorectal adenomas and carcinomas. Int J Cancer 2011, 129:2855-2866.

20. Tae CH, Ryu KJ, Kim SH, Kim HC, Chun HK, Min BH, Chang DK, Rhee PL, Kim $\mathrm{JJ}$, Rhee JC, Kim YH: Alcohol dehydrogenase, iron containing, 1 promoter hypermethylation associated with colorectal cancer differentiation. BMC cancer 2013, 13:142.

21. Eads CA, Lord RV, Kurumboor SK, Wickramasinghe K, Skinner ML, Long TI, Peters JH, DeMeester TR, Danenberg KD, Danenberg PV, Laird PW, Skinner KA: Fields of aberrant CpG island hypermethylation in Barrett's esophagus and associated adenocarcinoma. Cancer Res 2000, 60:5021-5026.

22. Eads CA, Lord RV, Wickramasinghe K, Long TI, Kurumboor SK, Bernstein L, Peters JH, DeMeester SR, DeMeester TR, Skinner KA, Laird PW: Epigenetic 
patterns in the progression of esophageal adenocarcinoma. Cancer Res 2001, 61:3410-3418.

23. Kwabi-Addo B, Chung W, Shen L, Ittmann M, Wheeler T, Jelinek J, Issa JP: Age-related DNA methylation changes in normal human prostate tissues. Clin Cancer Res 2007, 13:3796-3802.

24. Shen L, Kondo Y, Rosner GL, Xiao L, Hernandez NS, Vilaythong J, Houlihan PS, Krouse RS, Prasad AR, Einspahr JG, Buckmeier J, Alberts DS, Hamilton SR, Issa JP: MGMT promoter methylation and field defect in sporadic colorectal cancer. J Natl Canc Inst 2005, 97:1330-1338.

25. McClay JL, Aberg KA, Clark SL, Nerella S, Kumar G, Xie LY, Hudson AD, Harada A, Hultman CM, Magnusson PK, Sullivan PF, Van Den Oord EJ: A methylome-wide study of aging using massively parallel sequencing of the methyl-CpG-enriched genomic fraction from blood in over 700 subjects. Hum Mol Genet 2014, 23:1175-1185.

26. Derry MM, Raina K, Agarwal C, Agarwal R: Identifying molecular targets of lifestyle modifications in colon cancer prevention. Front Oncol 2013 3:119.

27. Bonsch D, Lenz B, Kornhuber J, Bleich S: DNA hypermethylation of the alpha synuclein promoter in patients with alcoholism. Neuroreport 2005, 16:167-170

28. Zhang $\mathrm{H}$, Herman $\mathrm{Al}$, Kranzler HR, Anton RF, Zhao H, Zheng W, Gelernter J: Array-based profiling of DNA methylation changes associated with alcohol dependence. Alcohol Clin Exp Res 2013, 37(Suppl 1):E108-E115.

29. Arzt E, Buric R, Stelzer G, Stalla J, Sauer J, Renner U, Stalla GK: Interleukin involvement in anterior pituitary cell growth regulation: effects of IL-2 and IL-6. Endocrinology 1993, 132:459-467.

30. Perry SW, Dewhurst S, Bellizzi MJ, Gelbard HA: Tumor necrosis factor-alpha in normal and diseased brain: conflicting effects via intraneuronal receptor crosstalk? J Neurovirol 2002, 8:611-624.

doi:10.1186/1471-2407-14-377

Cite this article as: Moon et al: Alcohol induces cell proliferation via hypermethylation of ADHFE1 in colorectal cancer cells. BMC Cancer 2014 14:377.

\section{Submit your next manuscript to BioMed Central and take full advantage of:}

- Convenient online submission

- Thorough peer review

- No space constraints or color figure charges

- Immediate publication on acceptance

- Inclusion in PubMed, CAS, Scopus and Google Scholar

- Research which is freely available for redistribution 